\title{
Simulated Annealing-Based Large-scale IP Traffic Matrix Estimation
}

\author{
Dingde Jiang, Xingwei Wang, Lei Guo and Zhengzheng Xu \\ Northeastern University \\ China
}

\section{Introduction}

Traffic matrix reflects the volume of traffic that flows between all pairs of sources and destinations in a network. Its element is referred to as an Origin-Destination (OD) pair (or flow). And traffic matrix gives network operators a global aspect of how all the traffic in a large-scale network flows. Thus, with traffic matrix as a key input of traffic engineering and network management, it is very important for network operators to accurately get the traffic matrix in a large-scale network. Unfortunately, as commented in (Papagiannaki et al., 2004), direct measurement of the traffic is not generally practical in the large-scale networks. In 1996, Vardi firstly introduced network tomography method to research the problem that traffic matrix in a network is indirectly measured. Since then, many researchers studied the problem and proposed many solutions(Cao et al., 2000; 2001; Juva, 2007; Soule et al., 2005; 2004; Tan \& Wang, 2007; Vardi, 1996; Zhang et al., 2003; 2005). Traffic matrix estimation is so far used by network operators to conduct the network management, network planning, traffic detecting and so on. However, since traffic matrix estimation holds the highly ill-posed properties (Soule et al., 2005; 2004; Tan \& Wang, 2007; Vardi, 1996; Zhang et al., 2003; 2005) and especially network traffic is a kind of nonstationary traffic (Cao et al., 2001), this subject is a challenging research problem.

The statistical inference techniques are first used to estimate traffic matrix over local area network (LAN). Authors in (Cao et al., 2000; Vardi, 1996) exploited the statistical model to model the OD flows in order to reduce the ill-posed nature of traffic matrix estimation. Zhang et al. (Zhang et al., 2003; 2005) introduced the gravity model into large-scale IP traffic matrix estimation. By the gravity model, they could obtain the prior information about OD flows and then successfully conduct the large-scale IP traffic matrix estimation. Nevertheless, as mentioned in (Juva, 2007; Soule et al., 2005), the statistical inference techniques are sensitive to the prior information, while the gravity model methods still have the larger estimation errors though it partially reduces the sensitivity to the prior information. Especially when the assumptions about OD flows hold, the gravity model methods are found to be more accurate than the statistical inference techniques, while their estimation accuracy decreases more quickly than that of the statistical inference techniques when the assumptions are not exactly true. Hence, this needs to develop a new method to estimate large-scale IP traffic matrix.

This chapter provides the reader with a method that large scale IP traffic matrix is estimated accurately by, as envisioned by the authors. It begins by explaining the need for using simulated annealing to estimate the traffic matrix, how traffic matrix estimation problem is defined, 
and possible motivation for using Simulated annealing to solve it. Then the chapter examines how our method is related to, but distinct from, previous work in traffic matrix estimation. The implementation of our method is discussed, and then simulation results and analysis are proposed. Finally, we present the important areas of future work and conclude our work to close the chapter.

\subsection{Problem Statement}

For a large-scale IP network, assuming there are the $n$ nodes and $L$ links, it will have the $N=n^{2}$ OD flows. Each of OD flows and link loads is time series. traffic matrix and link loads at time $t$ are denotes as $x(t)=\left(x_{1}(t), x_{2}(t), \ldots, x_{N}(t)\right)^{T}$ and $y(t)=\left(y_{1}(t), y_{2}(t), \ldots, y_{L}(t)\right)^{T}$ čňrespectively. In a large-scale IP network, traffic matrix $x(t)$ and link loads $y(t)$ are correlated by the $L$ by $N$ routing matrix $A=\left(A_{i j}\right)_{L \times N}$, where $A_{i j}$ is equal 1 if OD flow $j$ traverses link $i$, or zero. They follow the below constraints:

$$
y(t)=A x(t) .
$$

Link loads can be attained via the SNMP measurements. Routing matrix can be obtained by the status and configuration information of the network. Therefore, traffic matrix estimation is that, given link loads $y(t)$ and routing matrix $A$, one seeks to obtain a required solution $x(t)$ satisfied with (1). However, in the large-scale IP network, the number of OD flows is often by far larger than that of links, i.e. $L \ll N$. Eq. (1) denotes a highly under-constrained linear problem. It has the infinite solution for traffic matrix. Hence, large-scale IP traffic matrix estimation is a highly ill-posed inverse problem. How to overcome the ill-posed nature in this problem is the main challenge faced. Network tomography is a good way for this problem (Cao et al., 2000; Tebaldi \& West, 1998; Vardi, 1996; Zhang et al., 2003a).

In a large-scale IP network, traffic matrix and link loads vary with time, and are satisfied with the above linear relations. Previous studies show that OD flows, namely elements in traffic matrix, hold strongly daily pattern, and even weekly and monthly pattern. This suggests that OD flows hold the temporal correlations.

\subsection{Motivation and Requirements}

So far, simulated annealing method is studied extensively and has many successful solutions (Bryan et al., 2006; Hung et al., 2008; Tiwari \& Cosman, 2008; Thompson \& Bilbro, 2005). It is a non-numerical algorithm which is simple and globally optimal, and is suited well for solving the large-scale combinatorial optimization problems. However, because of the complexity of large-scale IP traffic matrix estimation, it is very difficult to use directly simulated annealing method to estimate it, and even it is not practical. And the solution space of traffic matrix is a continuous real subspace. Hence, due to the randomicity that conventional simulated annealing method generates a new solution, it is difficult to find quickly the globally optimal solution. To solve this problem and attain an accurate estimation of traffic matrix, we consider sufficiently spatial-temporal correlations of OD flows by using covariance matrix about OD flows, and combine partial flow measurement. By covariance matrix, one can quickly choose the direction in which the simulated annealing iterative process advances towards the globally optimal solution.

We investigate large-scale IP traffic matrix estimation problem and present a novel method called the simulated annealing and generalized inference (SAGI). Based on the conventional simulated annealing, we propose a modified simulated annealing process suited for largescale IP traffic matrix estimation. By the modified simulated annealing, we describe the traffic 
matrix estimation into a simulated annealing process. With the temperature dropping slowly, the traffic matrix's estimation gradually approaches to the true value. When temperature declines to the defined value, the estimation is attained. To obtain the accurate estimation, a heuristic method is introduced into the simulated annealing process by using covariance matrix about traffic matrix. Built on this heuristic way, the estimation can be quickly and accurately determined in the simulated annealing process. However, since traffic matrix estimation is a highly ill-posed problem, the estimation sought by simulated annealing may not precisely reflect the traffic matrix's inherent nature. We choose the Euclid and Mahalanobis distances as the optimal metric. This choice is based on the following reasons: (1) Euclid distance computes the whole distance of the vector. It deals with the difference between the elements of the vector in the identical way. (2) Mahalanobis distance can get rid of the disturbance of the correlations between the variables; it is not related with the measurement units; and Mahalanobis distance distinguishes the different characteristics between the variables. By combining these two different distances, a generalized inference is proposed to overcome further the traffic matrix's ill-posed nature. Hence, SAGI can accurately estimate large-scale IP traffic matrix. We use the real data from the Abilene (http:/ / www.cs.utexas.edu, 2004) and GÉANT (Uhlig et al., 2006) networks to validate SAGI. Simulation results show that SAGI exhibits the lower estimation errors and stronger robustness to noise.

\section{Related work}

Some papers have investigated traffic matrix estimation and proposed some solutions. Vardi (Vardi, 1996), Cao et al. (Cao et al., 2000), and Tebaldi et al. (Tebaldi \& West, 1998) used the statistical inference method to estimate traffic matrix only over the local area network. As mentioned in (Soule et al., 2005), these methods are sensitive to the prior, and estimation errors are larger. Medina et al. (Medina et al., 2002) showed that the basic assumptions based on the statistical models are not justified, and they also showed that, when their underlying assumptions are violated, the estimated results are bad. Furthermore, because these methods need to perform the complex mathematical computation, it takes some time to estimate traffic matrix. Hence, it is difficult to scale these methods to large-scale networks.

Zhang et al. (Zhang et al., 2003;a) discussed the problem of large-scale IP traffic matrix estimation by introducing the gravity model. Though, as mentioned in (Soule et al., 2005), their method partially reduced the sensitivity to the prior, it also has the larger errors, because it only considered the spatial correlations among the OD flows. Nucci et al. (Nucci et al., 2004) proposed the method that changed the under-constrained problem into the full rank one by changing the routing and then taking new SNMP measurements under this new routing map. Similarly, Soul et al. (Soule et al., 2004) presented a heuristic algorithm to compute the routing needed in order to obtain a full rank problem. Papagiannaki et al. (Papagiannaki et al., 2004) proposed a data-driven method that depends on measurements alone to obtain traffic matrix, without using the routing matrix and performing the inference, but based on measuring traffic matrix directly. Lakhina et al. (Lakhina et al., 2004) used the Principal Component Analysis to solve the traffic matrix estimation problem. Soule et al. (Soule et al., 2004) introduced the Kalman filtering into traffic matrix estimation. However, all the methods need to establish mathematical model about OD flows and perform the statistical inference, or combine the direct measurement of partial OD flows to infer traffic matrix. Thus, they need the complex mathematical computations. Different from the above methods, SAGI uses simulated annealing to handle large-scale IP traffic matrix estimation problem. By denoting traffic 
matrix estimation problem into simulated annealing process, we avoid complex mathematical computation and can attain the accurate estimation results.

Liang et al. (Liang et al., 2006), based on game theory, proposed a fast lightweight approach to OD flow estimation. Bermolen et al. (Bermolen et al., 2006) derived analytically the Fisher information matrix under the second moment statistics with the functional mean-variance relationship and then obtained the Cramer-Rao lower bound for the variance of traffic matrix estimator. By this bound, they could attain traffic matrix estimation. Juva (Juva, 2007) studied the sensitivity of the estimation accuracy to those underlying assumptions in the case of the gravity model based and second moment methods. They showed that if the assumptions hold, the gravity model based methods are more accurate, or their accuracy declines faster than that of the second moment methods. However, SAGI does not make any assumption about OD flows. It only sovle traffic matrix estimation problem with simulated annealing method. Due to the capacity of simulated annealing to solve the large-scale combinatorial optimization problems, SAGI is not sensitive to the assumption about OD flows and it is also robust to noise. Because the modified simulated annealing method is simple and fast, this makes it suited for handling the problem of the large-scale IP traffic matrix estimations.

\section{Implementation}

According to the characteristics of traffic matrix, multi-input and multi-output large-scale IP traffic matrix estimation model based on the modified simulated annealing method is presented in Figure 1, where $y(t)=\left(y_{1}(t), y_{2}(t), \ldots, y_{L}(t)\right)^{T}$ ( $L$ is the number of links in a network) is link loads at a particular time $t, x_{-} 0=\left(\hat{x}_{1}^{0}(t), \hat{x}_{x}^{0}(t), \ldots, \hat{x}_{N}^{0}(t)\right)^{T}(N$ is the number of OD flows in a network) the initial value of traffic matrix, $\hat{x}=\left(\hat{x}_{1}(t), \hat{x}_{x}(t), \ldots, \hat{x}_{N}(t)\right)^{T}$ the estimation of traffic matrix, $f$ cost function, and $x \_o p t, f \_$min, $x \_c u r, f \_c u r$ and $x \_g$ denotes optimal solution, minimum of cost function, current solution, current value of cost function and new solution in the iterative process of the modified simulated annealing method, respectively, and "Stop criterion" includes the maximum iteration steps and maximum unchanged times of cost function at a certain defined temperature. As showed in Figure 1, the model includes three parts: Initialing, Modified simulated annealing method and IPFP, where Initialing is used for initialing the variable that Modified simulated annealing method needs, Modified simulated annealing method for seeking globally optimal solution, and IPFP for satisfying the estimations of traffic matrix with tomographic constraints.

Generally, simulated annealing method includes generating new solution, computing cost function difference, ascertaining if new solution is accepted, and updating the iterative process. Now, according to the complexity of large-scale IP traffic matrix estimation, simulated annealing method is modified as follows.

\subsection{Cost Function}

According to Equation (1), cost function used for simulated annealing method is chosen as:

$$
f(x(t))=\|y(t)-A x(t)\|,
$$

where $\|$.$\| is L_{2}$ norm. Assume that the $k t h$ iterative result is $x^{k}(t)$, and the $(k+1)$ th iterative result is $x^{k+1}(t)$. And then cost function difference between the $k t h$ result and the $(k+1) t h$ one is denoted as:

$$
\Delta f^{k+1}=f\left(x^{k+1}(t)\right)-f\left(x^{k}(t)\right)
$$




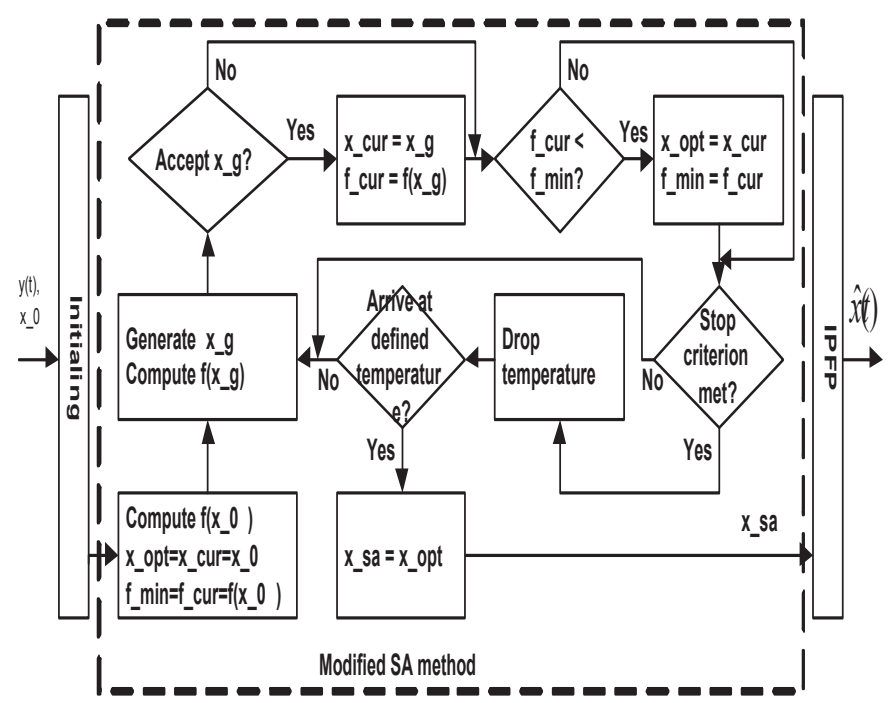

Fig. 1. Architecture representation of multi-input and multi-output large-scale IP traffic matrix estimation model based on the modified simulated annealing method, with the modified simulated annealing method showed in dotted line frame, IPFP used for adjusting the estimation of traffic matrix.

\subsection{Generating New Solution}

By generating randomly new solution, simulated annealing method makes current solution departing from locally optimal point to globally optimal point. However, the solution space of traffic matrix is a continuous real subspace. Hence, it is difficult of conventional simulated annealing method to find quickly the globally optimal solution. And so covariance matrix about OD flows is introduced to solve this problem.

Firstly, covariance matrix is attained, based on multivariate statistical analysis theory. Assume that traffic matrices of $S$ time plots, which are denoted as $X^{S}=(x(1), x(2), \ldots, x(S))$, are known. And then covariance matrix $C$ is represented by the following equation.

$$
\left\{\begin{array}{l}
U=\sum_{j=1}^{S}\left(x(i)-\overline{X^{S}}\right), \\
C=\frac{1}{S-1} U U^{T}, \\
\overline{X^{S}}=\frac{1}{S} \sum_{j=1}^{S} x(i) .
\end{array}\right.
$$

Equation (4) shows that main diagonal elements of covariance matrix $C$ represent the temporal correlations of traffic matrix sample, and other elements represent spatial-temporal correlations. As mentioned above, OD flows hold strongly daily pattern, and even weekly and monthly pattern, and so covariance matrix $C$ can be used to denote spatial-temporal correlations of traffic matrix. The following simulation results also show this.

According to the characteristics of large-scale IP traffic matrix, new solution in the simulated annealing process, from the $k t h$ iterative result $x^{k}(t)$ to the $(k+1) t h$ iterative result $x^{k+1}(t)$, is generated as follows.

$$
x^{k+1}(t)=x^{k}(t)+2 \times \text { rand } \times \Delta x^{k+1},
$$


where "rand" is the random number from 0 to 1 , and $\Delta x^{k+1}$ is a direction vector in the $(k+$ 1)th iterative process. It is a key problem to ascertain quickly $\Delta x^{k+1}$. The following uses the optimal method to attain it. To consider sufficiently spatial-temporal correlations of OD flows and satisfy the estimations of traffic matrix with tomographic constraints, select the objective function:

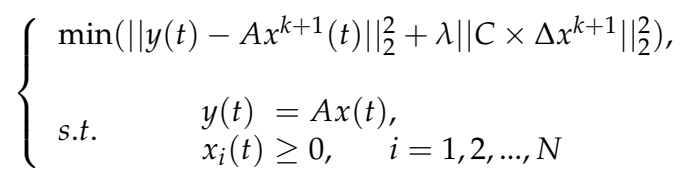

where $C$ is the covariance matrix denoted by equation (4), $\lambda$ a regularization parameter whose value is generally 0.01 or so.

Set

$$
x^{k+1}(t)=x^{k}(t)+\Delta x^{k+1},
$$

Substitute Equation (7) into Equation (6), and get the below objective function:

$$
\begin{cases}\min \left(\left\|y(t)-A\left(x^{k}(t)+\Delta x^{k+1}\right)\right\|_{2}^{2}+\lambda\left\|C \times \Delta x^{k+1}\right\|_{2}^{2}\right), & \\ \text { s.t. } \quad & y(t)=A x(t), \\ & x_{i}(t) \geq 0, \quad i=1,2, \ldots, N\end{cases}
$$

The least square solution of $(8)$ is

$$
\Delta x^{k+1}=\left(A^{T} A+\lambda \times C^{T} C\right)^{-1} A^{T}\left(y(t)-A x^{k}(t)\right),
$$

According to Equation (5) and (9), get the following new solution in the simulated annealing process.

$$
\left\{\begin{array}{l}
x^{k+1}(t)=x^{k}+2 \times \text { rand } \times \Delta x^{k+1} \\
\Delta x^{k+1}=\left(A^{T} A+\lambda \times C^{T} C\right)^{-1} A^{T} Y^{k} \\
Y^{k}=\left(y(t)-A x^{k}(t)\right) .
\end{array}\right.
$$

\subsection{Accepted Criterion}

The modified simulated annealing method uses Metroplis criterion to ascertain if new solution is accepted. Here, Metroplis criterion is denoted by the below equation.

$$
P\left(x^{k}(t) \Rightarrow x^{k+1}(t)\right)= \begin{cases}1 & \Delta f^{k+1} \leq 0, \\ \exp \left(\frac{\Delta f^{k+1}}{T}\right), & \Delta f^{k+1}>0 .\end{cases}
$$

where $T$ is the current temperature value. Equation (11) shows that when $\Delta f^{k+1} \leq 0$, new solution is accepted with probability value 1 , or with probability value $\exp \left(\frac{\Delta f^{k+1}}{T}\right)$.

\subsection{Updating the Iterative Process}

As showed in Figure 1, the modified simulated annealing method firstly computes cost function $f\left(x \_0\right)$ by the initial value $x_{-} 0$ of traffic matrix, and then generates new solution $x_{-} g$ in terms of Equation (10). If new solution is accepted, then update $x_{-}$cur and $f_{-}$cur, or if $f \_$cur $<f \_$min, update $x \_o p t$ and $f \_$min. Now if "Stop criterion" is not met, update the corresponding variables, generate the next new solution and repeat the above process, or drop temperature, and ascertain if temperature arrives at the defined value. If temperature arrives 
at the defined value, update $x \_s a=x \_o p t$. The two variables in the iterative process, namely $x_{-}$opt and $f \_$min, insure that the met optimal solution is not left out. Thus the modified simulated annealing method holds the memory capability.

Let $x^{0}(t), x_{-} o p t$, and $f_{-}$min denote the traffic matrix's prior value, optimal solution, minimum cost function in the iterative process of the simulated annealing, respectively. The traffic matrix's prior value is given by the last moment estimation $\hat{x}(t-1)$. This gives the below iterative equation:

$$
\begin{cases}x^{k+1}(t) & =x^{k}(t)+2 \times r \times \Delta x^{k+1} \\ x^{0}(t) & =\hat{x}(t-1) .\end{cases}
$$

Eqs. (4), (6), and (12) indicate that the modified simulated annealing has built the spatiotemporal model about traffic matrix. This model can accurately capture the traffic matrix's characteristics. However, as time advances forward, this model may not precisely capture the traffic matrix's properties and yields the estimation errors. As mentioned in (Soule et al., 2005), we use the partial flow measurement to calibrate this model. In addition, to follow the constraints in Eq. (6), iterative proportional fitting procedure (IPFP) is used to adjust the estimation obtained.

So far, we have proposed a modified simulated annealing method to attain the traffic matrix's estimation. The following Algorithm 1 proposes the complete steps of this method.

\section{Algorithm 1}

Step 1. Give the error $\varepsilon$, ratio factor $\alpha$, initial temperature $T_{0}$, minimum temperature $T_{\min }$, maximum iterative steps $K$, maximum unchanged times $M$ of cost function at certain temperature, and initial traffic matrix $x^{0}(t)$. And set temperature $T=T_{0}$, iterative step $k=0$, and the variable $m=0$. Then compute $f\left(x^{0}(t)\right)$ by Eq. (1), and set $x_{-}$opt $=x^{0}(t), f_{-} \min =f\left(x^{0}(t)\right)$. Step 2. Yield random value " $r$ " from 0 to 1. By Eq. (10), get new solution $x^{k+1}(t)$ and compute $f\left(x^{k+1}(t)\right)$.

Step 3. Compute cost function difference $\Delta f^{k+1}$ according to Eq. (3). If $\Delta f^{k+1}=0$, set $m=m+1$.

Step 4. Generate random value " $p_{-} r a n d "$ from 0 to 1 . If $\Delta f^{k+1} \leq 0$ or $\exp \left(\frac{\Delta f^{k+1}}{T}\right)>p_{-}$rand, accept new solution $x^{k+1}(t)$, or discard new solution $x^{k+1}(t)$.

Step 5. If $x^{k+1}(t)$ is accepted and $f\left(x^{k+1}(t)\right)<f_{-} \min$, update $x_{-} o p t=x^{k+1}(t)$ and $f_{-} \min =$ $f\left(x^{k+1}(t)\right)$, or If $x^{k+1}(t)$ is not accepted, $x^{k+1}(t)=x^{k}(t)$.

Step 6. If $k<K$ and $m<M$, set $k=k+1$ and go back to Step 2 .

Step 7. Drop temperature to $T=\alpha T$. if $T \geq T_{\min }$, set $k=0, m=0, x^{k}(t)=x_{-} o p t$, and go back to Step 2.

Step 8. Adjust $x_{-}$opt with IPFP and obtain the traffic matrix's estimation $\hat{x}_{s}(t)$.

Step 9. If $\left\|y(t)-A \hat{x}_{s}(t)\right\|_{2}^{2}<\varepsilon$, output the result, or directly measure the OD flows and go back to Step 1.

\subsection{Generalized Inference}

Since traffic matrix estimation is a highly ill-posed problem, how to seek to obtain a solution required is significantly difficult. Here we present a generalized inference method to further overcome the ill-posed nature of this problem. As mentioned in introduction, we choose the Euclid and Mahalanobis distance as the optimal metric. Thus, the objective function is given as follows: 


$$
\left\{\begin{array}{l}
\min \left((y(t)-A x(t))^{T} Q^{-1}(y(t)-A x(t))+\right. \\
\left.\|y(t)-A x(t)\|_{2}^{2}+\alpha\|D x(t)\|_{2}^{2}\right)
\end{array},\right.
$$

where $Q$ is the covariance matrix of link loads, $D$ denotes a smoothing matrix, and $\alpha$ represents a regularization parameter with its value being 0.01 or so.

Set

$$
x(t)=x_{0}(t)+\Delta x(t) .
$$

By Eqs. (13)-(14), obtain the least square solution:

$$
\begin{array}{r}
\Delta x(t)=\left(A^{T} A+A^{T} Q^{-1} A+\alpha D^{T} D\right)^{-1} \times \\
\left(A^{T}+A^{T} Q^{-1}\right)\left(y(t)-A x_{0}(t)\right) .
\end{array}
$$

Accordingly, attain the following iterative equation:

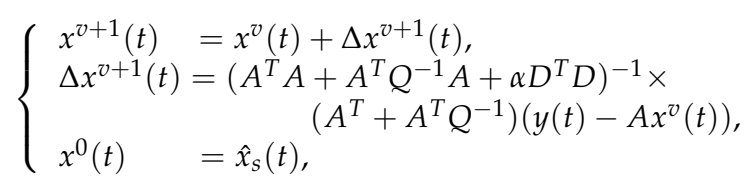

where $v$ represents the iterative step and $\hat{x}_{s}(t)$ is the estimation attained by simulated annealing. Eqs. (13)-(16) represent the generalized inference process. Algorithm 2 gives an overview of the generalized inference process proposed.

\section{Algorithm 2}

Step 1. Set the errors $\varepsilon$ and $\delta$, give iterative steps $V$, and let $v=0$ and $x^{0}(t)=\hat{x}_{s}(t)$.

Step 2. According to Eq. (16), perform the generalized inference process.

Step 3. If $\left\|y(t)-A x^{v+1}(t)\right\|_{2}^{2} \leq \varepsilon$ or $\left\|x^{v+1}(t)-x^{v}(t)\right\|_{2}^{2}$

$\leq \delta$ or $v>V$, exit and output the result, or go back Step 2 otherwise.

Up to now, we propose Algorithms 1 and 2 for the simulated annealing process and generalized inference, respectively. Below we summarize the complete SAGI method proposed.

Step 1. Obtain the traffic matrix $\hat{x}_{s}(t)$ according to Algorithm 1.

Step 2. By Algorithm 2, attain the optimization solution.

Step 3. Use IPFP to yield a more accurate estimation satisfied with the constraints in Eq. (13).

\section{Simulation Results and Analysis}

We conduct a series of simulations to validate SAGI, analyzing traffic matrix estimation errors (spatial relative errors (SREs) and temporal relative errors (TREs)) and robustness. Since TomoGravity (Zhang et al., 2003; 2005) and 1-Inverse (Tan \& Wang, 2007) is reported as the accurate methods of traffic matrix estimation, SAGI will be compared with them. We use, respectively, the ten-day and twenty-one-day real data from the Abilene (http:/ / www.cs.utexas.edu, 2004) and GÉANT (Uhlig et al., 2006) network to simulate the performance of three methods. The first seven-day and fourteen-day data from the Abilene and GÉANT network are, respectively, used to construct the covariance matrix according to Eq. (4), while the rest are exploited to test three methods.

The SREs and TREs are denoted as follows: 


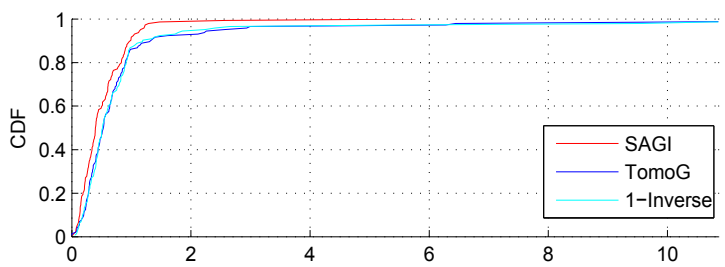

(a) $x=$ L2 norm, Spatial relative errors

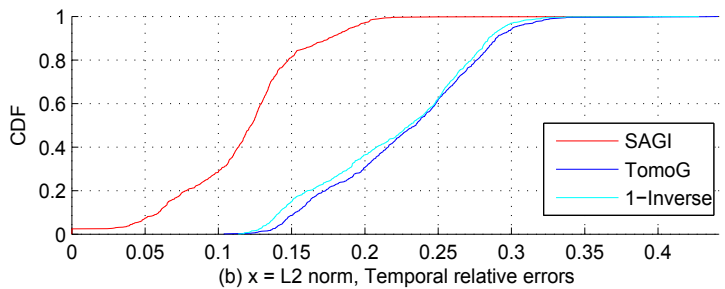

Fig. 2. CDF of spatial and temporal relative errors in Abilene.

$$
\left\{\begin{array}{l}
\operatorname{err}_{s p}(n)=\frac{\left\|\hat{x}_{T}(n)-x_{T}(n)\right\|_{2}}{\left\|x_{T}(n)\right\|_{2}}, \\
\operatorname{err}_{t m}(t)=\frac{\left\|\hat{x}_{N}(t)-x_{N}(t)\right\|_{2}}{\left\|x_{N}(t)\right\|_{2}}, \\
n=1,2, \ldots, N ; \quad t=1,2, \ldots, T
\end{array}\right.
$$

where $N$ and $T$ are the total number of OD flows and measurement moments, respectively; $\|.\|_{2}$ is $L_{2}$ norm; $\operatorname{err}_{s p}(n)$ and $\operatorname{err}_{t m}(t)$ denote the SREs and TREs, respectively. To precisely evaluate the estimation performance of three methods, we examine the cumulative distribution functions (CDFs) of their SREs and TREs. Figs.1 and 2 plot their CDFs in the Abilene and GÉANT network, respectively.

From Figs.1 and 2, we can see that the curves of the SREs' and TREs' CDFs of TomoGravtiy and $\{1\}$-Inverse are far below those of SAGI, while TomoGravtiy's and $\{1\}$-Inverse's are close. Furthermore, In Fig.1a, for SAGI, about 79\% of OD flows are tracked with SREs less than 0.8 , while less than $74 \%$ for TomoGravity and less than $71 \%$ for $\{1\}$-Inverse. In Fig.2a, for SAGI, about $86 \%$ of OD flows are tracked with SREs less than 0.8 , while about $26 \%$ for TomoGravity and about 35\% for 1-Inverse. This shows that, in Abilene and GÉANT network, the spatial estimation errors of SAGI are far lower than those of other two methods, while those of TomoGravity and $\{1\}$-Inverse are close. Analogously, we can also see that, in Fig.1b, about $97 \%$ of measurement moments, for SAGI, can be tracked with TREs less than 0.2 , while $30.6 \%$ for TomoGravity and $36.4 \%$ for $\{1\}$-Inverse. In Fig. $2 b$, SAGI can track $94 \%$ of measurement moments with TREs less than $10.12 \%$, while TomoGravity with TREs less than $50.28 \%$ and $\{1\}$-Inverse with TREs less than $53.67 \%$. This indicates that in Abilene and GÉANT network, the temporal estimation errors of SAGI are far lower than those of other two methods. However, TomoGravity's is lower than $\{1\}$-Inverse's in Abilene network, while $\{1\}$-Inverse's is lower than TomoGravity's in GÉANT network. Hence, SAGI can more accurately estimate traffic matrix than TomoGravity and $\{1\}$-Inverse. 


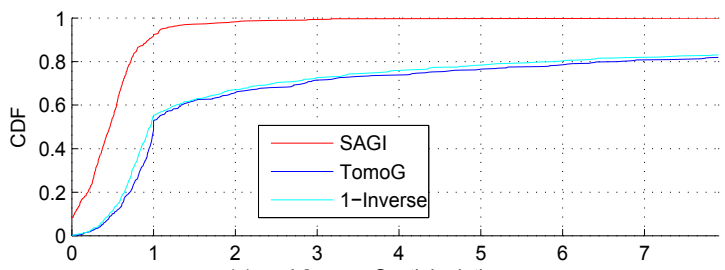

(a) $x=$ L2 norm, Spatial relative errors

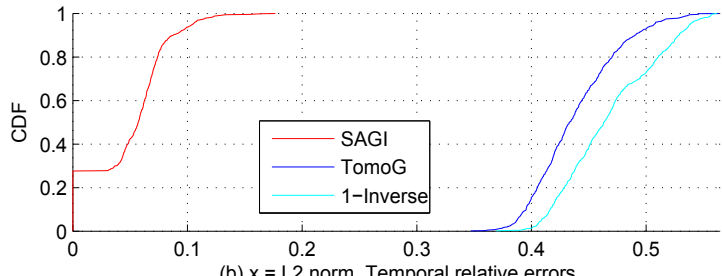

Fig. 3. CDF of spatial and temporal relative errors in GÉANT.

To evaluate the impact of noise on three methods, we introduce an error term $\theta(t)$ to (1) and attain the equation $y_{n}(t)=A x(t)+\theta(t)$, where $\theta(t)=y_{n}(t) \times \eta(0, \zeta)$, and $\eta(0, \zeta)$ denotes a normal distribution with zero mean and standard deviation. We discuss the robustness of three methods in three cases: $\zeta=0.01, \zeta=0.03$, and $\zeta=0.05$. We use the following spatial root mean squared relative error (SRMSRE) and temporal root mean squared relative error (TRMSRE) to evaluate the robustness of three methods in the Abilene and GÉANT network.

$$
\left\{\begin{array}{l}
\text { SRMSRE }=\frac{1}{N} \sum_{n=1}^{N} \frac{\left\|\hat{x}_{T}(n)-x_{T}(n)\right\|_{2}}{\left\|x_{T}(n)\right\|_{2}}, \\
\text { TRMSRE }=\frac{1}{T} \sum_{t=1}^{T} \frac{\left\|\hat{x}_{N}(t)-x_{N}(t)\right\|_{2}}{\left\|x_{N}(t)\right\|_{2}} .
\end{array}\right.
$$

Tables 1 and 2 show the impact of noise on three methods in the Abilene and GÁENT network, respectively. From both tables, we can see that, in three cases: $\zeta=0.01, \zeta=0.03$, and $\zeta=0.05$, the SRMSRE's and TRMSRE's changes of SAGI are lower than those of other two methods. Hence, SAGI is more robust to noise.

\section{Future Questions and Research Areas}

The previous sections make an investigation for how simulated annealing method is exploited to solve the large-scale traffic matrix estimation. We now examine major issues of the related research for future. With the wireless sensor networks and the Internet of things advancing, IP networks will become more ubiquitous and heterogeneous. Though this chapter exploits the simulated annealing to be able to make the accurate estimation for traffic matrix, SAGI method is only validated in the large-scale IP backbone networks. We do not know if this method is suited for the ubiquitous and heterogeneous networks either. Hence, it is necessary to validate SAGI method in the ubiquitous and heterogeneous networks.

Additionally, there is an implicit assumption in this chapter that the topology of the IP networks keeps up unchanged. In fact, the topology of the networks will change with the envi- 
ronment of networks, for example, when the fault of the networks takes place, their topologies will vary correspondingly. Network topology is related to route matrix. Thus we also consider if SAGI can still estimate accurately traffic matrix when network topology changes.

Table 1. Impact of noise on three methods in Abilene

\begin{tabular}{c|c|c|c|c}
\hline \multicolumn{2}{c|}{ Noise Level } & $\delta=0.01$ & $\delta=0.03$ & $\delta=0.05$ \\
\hline \hline \multirow{2}{*}{$\begin{array}{c}\text { Link } \\
\text { loads }\end{array}$} & SRMSRE & $1.00 \%$ & $3.01 \%$ & $5.05 \%$ \\
\cline { 2 - 5 } & TRMSRE & $0.98 \%$ & $2.97 \%$ & $4.96 \%$ \\
\hline \multirow{2}{*}{ SAGI } & SRMSRE & $53.10 \%$ & $53.85 \%$ & $55.03 \%$ \\
\cline { 2 - 5 } & TRMSRE & $13.93 \%$ & $14.22 \%$ & $14.75 \%$ \\
\hline \multirow{2}{*}{ TomoG } & SRMSRE & $96.79 \%$ & $99.39 \%$ & $104.66 \%$ \\
\cline { 2 - 5 } & TRMSRE & $22.82 \%$ & $23.48 \%$ & $24.91 \%$ \\
\hline \multirow{2}{*}{$\begin{array}{c}\{1\}- \\
\text { Inverse }\end{array}$} & SRMSRE & $99.61 \%$ & $101.76 \%$ & $105.76 \%$ \\
\cline { 2 - 5 } & TRMSRE & $22.22 \%$ & $22.87 \%$ & $24.09 \%$ \\
\hline \hline
\end{tabular}

Table 2. Impact of noise on three methods in GÉANT

\begin{tabular}{c|c|c|c|c}
\hline \multicolumn{2}{c|}{ Noise Level } & $\delta=0.01$ & $\delta=0.03$ & $\delta=0.05$ \\
\hline \hline \multirow{2}{*}{$\begin{array}{c}\text { Link } \\
\text { loads }\end{array}$} & SRMSRE & $0.98 \%$ & $2.95 \%$ & $5.00 \%$ \\
\cline { 2 - 5 } & TRMSRE & $0.99 \%$ & $2.97 \%$ & $5.02 \%$ \\
\hline \multirow{2}{*}{ SAGI } & SRMSRE & $87.55 \%$ & $88.30 \%$ & $89.38 \%$ \\
\cline { 2 - 5 } & TRMSRE & $29.05 \%$ & $29.65 \%$ & $30.85 \%$ \\
\hline \multirow{2}{*}{ TomoG } & SRMSRE & $139.03 \%$ & $141.34 \%$ & $144.61 \%$ \\
\cline { 2 - 5 } & TRMSRE & $42.85 \%$ & $44.13 \%$ & $46.51 \%$ \\
\hline \multirow{2}{*}{$\begin{array}{c}\{1\}- \\
\text { Inverse }\end{array}$} & SRMSRE & $137.99 \%$ & $139.89 \%$ & $143.53 \%$ \\
\cline { 2 - 5 } & TRMSRE & $45.85 \%$ & $47.05 \%$ & $49.27 \%$ \\
\hline \hline
\end{tabular}

\section{Conclusion}

This chapter has proposed a new method to estimate large-scale IP traffic matrix. By describing the traffic matrix estimation into a modified simulated annealing process and using the generalized inference, we have successfully overcome its ill-posed nature. Simulation results on both real networks show that SAGI exhibits the lower estimation errors and stronger robustness to noise. Although traffic matrix estimation is becoming more and more important, the accurate estimation for it is not becoming increasingly easier. Based on the simulated annealing method, this chapter manages to attain accurately the large-scale IP traffic matrix estimation by considering the spatial and temporal correlations.

\section{References}

Bryan, K., Cunningham, P., and Bolshakova, N., "Application of simulated annealing to the bi-clustering of gene expression data", IEEE Trans. Inf. Technol. Biomed., Vol. 10, No. 3, pp. 519-525, 2006.

Bermolen P, Vaton S, Juva I. Search for optimality in traffic matrix estimation: A rational approach by cramlerr-rao lower bounds. In: Proceedings of the 2nd EuroNGI NGI Conf. on Next Generation Internet Design and Engineering; 2006. p. 224-31.

Cao, J.; Davis, D.; Weil, S.; Yu, B. Time-varying network tomography. J. Amer. Stat. Assoc. 2000; 95:1063-75. 
Cao, J.; Cleveland, W.; Lin, D. et al. On the nonstationarity of internet traffic. In: Proceedings of ACM SIGMETRICS'01. Cambridge, MA, 2001. p. 102-12.

Hung, M.; Ho, S.; Shu, L.; Hwang, S.; and Ho, S.-Y. "A Novel Multiobjective Simulated Annealing Algorithm for Designing Robust PID Controllers", IEEE Trans. on Systems, Man, and Cybernetics-Part A, Systems and Humans, Vol. 38, No. 2 , pp. 319-330, MARCH. 2008.

http://www.cs.utexas.edu/ yzhang/research/abilene-tm/.

Juva, I. Sensitivity of traffic matrix estimation techniques to their underlying assumption. In: Proceedings of ICC'07. Glasgow, Scotland, 2007. p. 562-8.

Jiang, D.; Wang, X.; and Lei, G. An optimization method of large-scale IP traffic matrix estimation. AEU-International Journal of Electronics and Communications, 2009, In Press.

Jiang, D.; Wang, X.; Lei, G. et al. Accurate estimation of large-scale IP traffic matrix. AEUInternational Journal of Electronics and Communications, 2009, In Press.

Jiang, D. and Hu, G. Large-scale IP traffic matrix estimation based on simulated annealing. In Proceedings of the IEEE International Conference on Communications Systems (ICCS'08), Guangzhou, China, 19-21 November. 2008, 1-4.

Lakhina A, Papagiannaki K, Crovella M, et al. Structural analysis of network traffic flows. In Proceedings of ACM Sigmetrics, New York, June 2004.

Liang G, Taft N, Yu B. A fast lightweight approach to origin-destination ip traffic estimation using partial measurements. IEEE Transactions on Information Theory 2006; 52(6): 2634-48.

Medina A, Taft N, Salamatian K, et al. Traffic matrix estimation: Existing techniques and new directions. In Proceedings of ACM SIGCOMM'02, Pittsburgh, USA, August 2002.

Nucci A, Cruz R, Taft N, et al. Design of IGP link weight changes for estimation of traffic matrices. In Proceedings of IEEE Infocom, Hong Kong, March 2004.

Papagiannaki, K.; Taft, N.; and Lakhina, A. A distributed approach to measure traffic matrices. In: Proceedings of ACM Internet Measurement Conference (IMC'04). Taormina, Italy, 2004. p. 161-74.

Soule, A.; Lakhina, A.; Taft, N. et al. Traffic matrices: balancing measurements, inference and modeling. In: Proceedings of ACM SIGMETRICS'05. Banff, Canada, 2005. p.362-73,

Soule, A.; Salamatian, K.; Nucci, A.; and Taft, N.Traffic Matrix tracking using Kalman Filtering. LIP6 Research Report RP-LIP6-2004-07-10, LIP6, 2004.

Soule A, Nucci A, Leonardi E, et al. How to identify and estimate the largest traffic matrix elements in a dynamic environment. In Proceedins of ACM Sigmetrics, New York, June 2004.

Tan, L. and Wang, X. A novel method to estimate IP traffic matrix. IEEE Communications Letters 2007;11: 907-9.

Tebaldi, C. and West, M. "Bayesian inference on network traffic using link count data," Journal of the American Stati-stical Association, vol. 93, no. 442, pp. 557-576, June 1998.

Tiwari, M. and Cosman, P. C. "Selection of Long-Term Reference Frames in Dual-Frame Video Coding Using Simulated Annealing", IEEE Signal Processing Letters. Vol. 15, pp. 249$252,2008$.

Thompson, D. R. and Bilbro, G. L. "Sample-sort simulated annealing", IEEE Transactions on Systems, Man, and Cybernetics - Part B: Cybernetics, vol. 35, no. 3, pp. 625-632, June 2005.

Uhlig S, Quoitin B, Balon S, et al. Providing public intradomain traffic matrices to the research community. ACM SIGCOMM Computer Communication Review 2006;36:83-6 
Vardi, Y. Network tomography: estimating source-destination traffic intensities from link data. J. Amer. Stat. Assoc. 1996; 91(433): 365-377.

Zhang ,Y.; Roughan, M.; Duffield, N.; and Greenberg, A. Fast accurate computation of largescale IP traffic matrices from link loads. In: Proceedings of ACM SIGMETRICS'03. San Diego, California, USA, 2003. p. 206-17.

Zhang, Y.; Roughan, M.; Lund, C.; Donoho, D. Estimating point-to-point and point-tomultipoint traffic matrices: an information-theoretic approach. IEEE/ACM Trans Networking 2005; 13:947-60.

Zhang, Y.; Roughan, M.; Lund, C.; and Donoho, D. "An Information Theoretic Approach to Traffic Matrix Estimation," In ACM SIGCOMM, Karlsruhe, Germany, August 2003. 
Simulated Annealing.

Theory with Applications

estes by hui chibants

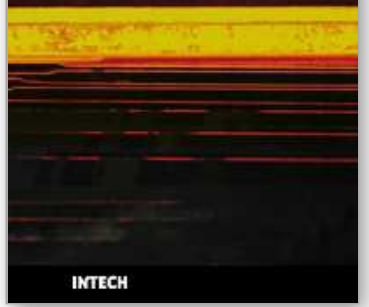

\section{Simulated Annealing, Theory with Applications}

Edited by Rui Chibante

ISBN 978-953-307-134-3

Hard cover, 292 pages

Publisher Sciyo

Published online 18, August, 2010

Published in print edition August, 2010

The book contains 15 chapters presenting recent contributions of top researchers working with Simulated Annealing (SA). Although it represents a small sample of the research activity on SA, the book will certainly serve as a valuable tool for researchers interested in getting involved in this multidisciplinary field. In fact, one of the salient features is that the book is highly multidisciplinary in terms of application areas since it assembles experts from the fields of Biology, Telecommunications, Geology, Electronics and Medicine.

\section{How to reference}

In order to correctly reference this scholarly work, feel free to copy and paste the following:

Dingde Jiang, Xingwei Wang, Lei Guo and Zhengzheng Xu (2010). Simulated Annealing-Based Large-Scale IP Traffic Matrix Estimation, Simulated Annealing, Theory with Applications, Rui Chibante (Ed.), ISBN: 978-953307-134-3, InTech, Available from: http://www.intechopen.com/books/simulated-annealing--theory-withapplications/simulated-annealing-based-large-scale-ip-traffic-matrix-estimation

\section{INTECH}

open science | open minds

\section{InTech Europe}

University Campus STeP Ri

Slavka Krautzeka 83/A

51000 Rijeka, Croatia

Phone: +385 (51) 770447

Fax: +385 (51) 686166

www.intechopen.com

\section{InTech China}

Unit 405, Office Block, Hotel Equatorial Shanghai

No.65, Yan An Road (West), Shanghai, 200040, China

中国上海市延安西路65号上海国际贵都大饭店办公楼 405 单元

Phone: +86-21-62489820

Fax: +86-21-62489821 
(C) 2010 The Author(s). Licensee IntechOpen. This chapter is distributed under the terms of the Creative Commons Attribution-NonCommercialShareAlike-3.0 License, which permits use, distribution and reproduction for non-commercial purposes, provided the original is properly cited and derivative works building on this content are distributed under the same license. 